\title{
Orbital magnetization in periodic insulators
}

\author{
T. Thonhauser, ${ }^{1}$ Davide Ceresoli, ${ }^{2}$ David Vanderbilt,${ }^{1}$ and R. Resta ${ }^{3}$ \\ ${ }^{1}$ Department of Physics and Astronomy, Rutgers University, Piscataway, New Jersey 08854, USA \\ ${ }^{2}$ International School for Advanced Studies (SISSA/ISAS) and INFM-DEMOCRITOS, Trieste, Italy \\ ${ }^{3}$ Dipartimento di Fisica Teorica Università di Trieste and INFM-DEMOCRITOS, Trieste, Italy
}

(Dated: October 25, 2018)

\begin{abstract}
Working in the Wannier representation, we derive an expression for the orbital magnetization of a periodic insulator. The magnetization is shown to be comprised of two contributions, an obvious one associated with the internal circulation of bulk-like Wannier functions in the interior, and an unexpected one arising from net currents carried by Wannier functions near the surface. Each contribution can be expressed as a bulk property in terms of Bloch functions in a gauge-invariant way. Our expression is verified by comparing numerical tight-binding calculations for finite and periodic samples.

PACS numbers: 75.10.-b, 75.10.Lp, 73.20.At, 73.43.-f
\end{abstract}

Recent years have seen a surge of interest in issues of charge and spin transport in magnetic materials and nanostructures, notably the development of a theory of the intrinsic anomalous Hall conductivity and some controversies surrounding the spin-Hall effect [1]. In this context it is quite surprising that the theory of orbital magnetization, essential for any proper description of magnetism, has remained in a primitive state. Linearresponse methods allow calculations of magnetization changes [2-5], but not of the magnetization itself.

Hirst [6] has emphasized that a knowledge of the bulk local current density $\mathbf{J}(\mathbf{r})$ is insufficient, even in principle, to determine the macroscopic orbital magnetization $\mathbf{M}$, just as the density $\rho(\mathbf{r})$ cannot be used to determine the electric polarization $\mathbf{P}$. Thus, the theory of $\mathbf{M}$ today is in a condition very similar to that of $\mathbf{P}$ in the early 1990s, when the problem of computing finite polarization changes was solved by the introduction of the Berry-phase theory $[7,8]$. The essential difficulty, that the matrix elements of the position operator $\mathbf{r}$ are not well-defined in the Bloch representation, could be overcome by reformulating the problem in the Wannier representation. Because Wannier functions (WFs) are exponentially localized in an insulator, matrix elements of $\mathbf{r}$ between WFs are indeed well-defined.

Here we show that it is possible to formulate a corresponding theory of the orbital magnetization for an insulator with broken time-reversal symmetry. The problem is analogous, with the circulation operator $\mathbf{r} \times \mathbf{v}$ now being ill-defined in the Bloch representation. Working instead in the Wannier representation, we write the orbital magnetization as a gauge-invariant Brillouin-zone integral over occupied Bloch functions. It contains two terms, the first of which describes the internal circulation of bulk-like WFs [9]. The second is much more subtle, arising only from surface WFs and reflecting the fact that the information about surface currents needed to define the macroscopic magnetization is actually contained in the bulk bandstructure. The resulting formula is consis- tent with a recent semiclassical argument [10] and can easily be implemented in existing first-principles codes.

For our derivation, we restrict ourselves to the case of an insulator described by a one-particle Hamiltonian with broken time-reversal symmetry. While the restriction to insulators is essential for the theory of polarization, we suspect that it is less so here, so that future generalizations to metals are not ruled out. We also require a vanishing macroscopic magnetic field (or, more generally, an integer number of flux quanta per unit cell) so that the Bloch wavevector $\mathbf{k}$ remains a good quantum number. We have in mind cases in which a staggered magnetic field averages to zero over the unit cell, or in which the time-reversal breaking comes about through spin-orbit coupling to a background of ordered local moments [11-15]. For simplicity we work with spinless electrons (the generalization to the spin-unrestricted case being straightforward) and furthermore restrict ourselves to zero-Chern-number insulators $[11,12]$.

Let us consider a finite sample representing a fragment of a larger crystalline system. We assume that the occupied states can be represented in terms of well-localized orthonormal orbitals $\left|\phi_{i}\right\rangle$, which we will refer to as Wannier functions. If we introduce the velocity operator as

$$
\mathbf{v}=-\frac{i}{\hbar}[\mathbf{r}, H]
$$

then the total magnetic moment of the finite system involves the matrix elements $\left\langle\psi_{i}|\mathbf{r} \times \mathbf{v}| \psi_{i}\right\rangle$, where the $\left|\psi_{i}\right\rangle$ are the occupied eigenstates of $H$. By invariance of the trace, this can be written in terms of WFs as

$$
\mathbf{m}=-\frac{e}{2 c} \sum_{i}\left\langle\phi_{i}|\mathbf{r} \times \mathbf{v}| \phi_{i}\right\rangle,
$$

where $-e$ is the electron charge. The magnetization $\mathbf{M}$ can then be defined as the magnetic moment $\mathbf{m}$ per unit volume. For large but finite samples, all $\left|\phi_{i}\right\rangle$ that are sufficiently far from the surface become exponentially similar to bulk WFs, which we will denote 
(a)

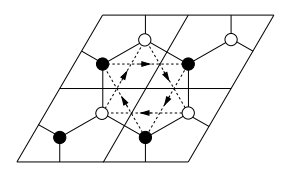

(b)

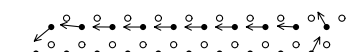

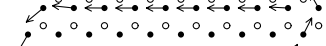

$0^{\circ} \cdot 0^{\circ} \cdot 0^{\circ} \cdot 0^{\circ}, 0^{\circ} \cdot 0$

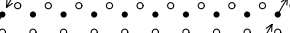

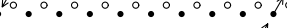

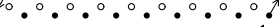

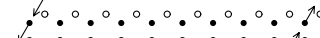

$\therefore \circ \therefore \circ \therefore \circ$

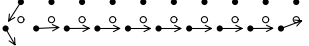

FIG. 1: (a) Four unit cells of the Haldane model. Filled (open) circles denote sites with $E_{0}=-2(+2)$. Arrows indicate sign of the phase $\varphi$ for second-neighbor hopping. (b) Net currents $-e \mathbf{v}_{i}$ associated with WFs, plotted at their centers $\overline{\mathbf{r}}_{i}$, for a $10 \times 10$ sample with $\varphi=\pi / 4$. Currents decrease rapidly into the bulk, so that only surface currents are visible.

as $\left|w_{i}\right\rangle$. For the electric polarization, the transition $\left\langle\phi_{i}|\mathbf{r}| \phi_{i}\right\rangle \rightarrow\left\langle w_{i}|\mathbf{r}| w_{i}\right\rangle$ describes the polarization of periodic systems correctly [7]. Thus, it is tempting to assume that the magnetization should be expressible in a similar way in terms of the circulation $\left\langle w_{i}|\mathbf{r} \times \mathbf{v}| w_{i}\right\rangle$ of bulk WFs.

We set out to verify this hypothesis in the context of numerical tight-binding calculations. For simplicity, we chose the Haldane model [11], which is comprised of a honeycomb lattice with two tight-binding sites per cell with site energies $\pm E_{0}$, real first-neighbor hoppings $t_{1}$, and complex second-neighbor hoppings $t_{2} e^{ \pm i \varphi}$ as shown in Fig. 1(a). For our tests, we have chosen a lattice constant equal to unity, $E_{0}= \pm 2, t_{1}=1$ and $t_{2}=1 / 3$ and allowed $\varphi$ to vary [16]. We treat the upper band as empty and the lower band as occupied. The corresponding WFs were obtained by acting with the band projector on a set of $\delta$-functions located on the sites with $E_{0}=-2$ and applying a subsequent symmetric orthonormalization.

The results of our numerical calculations for a finite $30 \times 30$ sample are depicted as the symbols in Fig. 2 . First, we calculated the total magnetic moment according to Eq. (2) and divided by the total sample area $A$ to obtain the magnetization $M$ indicated as circles in Fig. 2. Next, we evaluated the contribution to Eq. (2) from a single WF deep in the bulk of the finite sample and divided by the unit-cell area $A_{0}$ to obtain a "local circulation" magnetization $M_{\mathrm{LC}}$ plotted as triangles in Fig. 2. We expected these two quantities to agree with each other within some numerical tolerance. On the contrary, the results indicate no agreement whatsoever.

This surprising result forced us to reconsider our entire line of argument, revealing a profound oversight. It is easily shown that each bulk band of an insulating crystal must carry no net current, even in the absence of timereversal symmetry. This means that each bulk-like WF, such as the one that we chose from the deep interior of the sample, must carry no net current, as is easily confirmed in the numerical calculation. We had assumed that the WFs at the boundary of the finite sample would likewise carry no net current, but this assumption is incorrect. In fact, the WFs near the boundary do carry a net current,

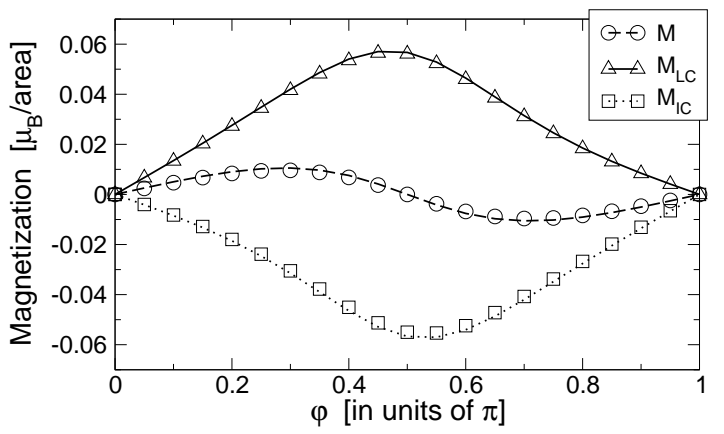

FIG. 2: Numerical results for the magnetization (in Bohr magnetons per unit area) for the Haldane model. Symbols denote results from a finite $30 \times 30$ sample, while curves represent results of a k-space calculation on a periodic system.

and the total circulation associated with these net currents provides just the needed contribution to resolve the discrepancy.

This is illustrated in Fig. 1(b), where we have plotted the net current $-e \mathbf{v}_{i}=-e\left\langle\phi_{i}|\mathbf{v}| \phi_{i}\right\rangle$ located at the Wannier center $\overline{\mathbf{r}}_{i}=\left\langle\phi_{i}|\mathbf{r}| \phi_{i}\right\rangle$ for each WF in the sample. While confirming that the net currents in the deep interior are exponentially small, the results reveal that the WFs near the surface do carry a substantial current that contributes extensively to the total magnetic moment of the sample. Dividing by the sample area, we obtain an "itinerant circulation" contribution $M_{\text {IC }}$ to the magnetization that is plotted as squares in Fig. 2. A glance at the figure suggests, and numerical tests confirm, that $M=M_{\mathrm{LC}}+M_{\mathrm{IC}}$ within numerical precision [17].

It is not surprising that $M_{\mathrm{LC}}$, corresponding to the local circulation of a bulk WF, is a bulk property of the insulator [9]. It is far less clear whether $M_{\mathrm{IC}}$ is also a bulk property. To show that it is, we go back to Eq. (2). For simplicity, we restrict ourselves henceforth to the case of a two dimensional system with a single occupied band (the generalization to three dimensions is straightforward, while the multiband treatment is more subtle). Then Eq. (2) can be rewritten as

$$
M=-\frac{e}{2 A c} \sum_{i}[\underbrace{\left\langle\phi_{i}\left|\left(\mathbf{r}-\overline{\mathbf{r}}_{i}\right) \times \mathbf{v}\right| \phi_{i}\right\rangle}_{\text {LC }}+\underbrace{\overline{\mathbf{r}}_{i} \times\left\langle\phi_{i}|\mathbf{v}| \phi_{i}\right\rangle}_{\mathrm{IC}}],
$$

where again 'LC' and 'IC' correspond to local and itinerant circulation contributions, respectively. We divide the finite sample into an "interior" and a "surface" region in such a way that the latter occupies a non-extensive fraction of the total sample area in the thermodynamic limit. We label WFs from the surface and interior regions as $\left|\phi_{s}\right\rangle$ and $|\mathbf{R}\rangle$, respectively, where $\mathbf{R}$ is a lattice vector.

Next, we note that the WFs in the surface region make a negligible contribution to the LC term of Eq. (3) since they occupy a non-extensive fraction of the area in the 


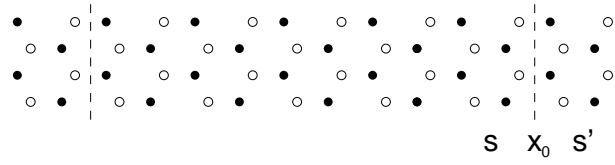

FIG. 3: Horizontal slice from a sample that extends indefinitely in the vertical direction but is otherwise similar to the one in Fig. 1. Vertical dashed lines delimit bulk and surface regions in which WFs are labeled by $s$ and $s^{\prime}$, respectively.

thermodynamic limit. The LC term then becomes

$$
\begin{aligned}
M_{\mathrm{LC}} & =-\frac{e}{2 A c} \sum_{\mathbf{R}}\left\langle\mathbf{R}\left|\left(\mathbf{r}-\overline{\mathbf{r}}_{\mathbf{R}}\right) \times \mathbf{v}\right| \mathbf{R}\right\rangle \\
& =-\frac{e}{2 A_{0} c}\langle\mathbf{0}|\mathbf{r} \times \mathbf{v}| \mathbf{0}\rangle .
\end{aligned}
$$

Here we have used translational symmetry and the fact that $\langle\mathbf{R}|\mathbf{v}| \mathbf{R}\rangle=0$ (since bulk bands carry no net current). Eq. (4) shows that $M_{\mathrm{LC}}$ can be expressed simply in terms of the bulk WF $|\mathbf{0}\rangle$ in the home unit cell. Turning to the IC term, the interior WFs now make no contribution (again because $\langle\mathbf{R}|\mathbf{v}| \mathbf{R}\rangle=0$ ) so that

$$
M_{\mathrm{IC}}=-\frac{e}{2 A c} \sum_{s} \overline{\mathbf{r}}_{s} \times \mathbf{v}_{s}
$$

where the sum runs over surface WFs only.

We now concentrate on this IC contribution and consider a vertical strip of which one horizontal section is sketched in Fig. 3, and choose vertical boundaries (dashed lines) to discriminate between interior and surface regions. Focusing on the right edge, we use labels $s$ and $s^{\prime}$ to label WFs in the interior $\left(x<x_{0}\right)$ and surface $\left(x>x_{0}\right)$ regions, respectively. The vertical macroscopic current flowing in the right surface is

$$
I_{y}=-\frac{e}{\Delta l} \sum_{s^{\prime}}^{\prime} v_{s^{\prime}, y}
$$

where the primed sum is further limited to WFs whose centers are inside a vertical segment of length $\Delta l$, equal to the vertical repeat unit. The current carried by the $i$-th WF can be written in terms of contributions from its neighbors as

$$
\mathbf{v}_{i}=-\frac{i}{\hbar}\left\langle\phi_{i}|[\mathbf{r}, H]| \phi_{i}\right\rangle=\sum_{j} \mathbf{v}_{\langle j i\rangle}
$$

where $\mathbf{v}_{\langle j i\rangle}=(2 / \hbar) \operatorname{Im} \mathbf{r}_{i j} H_{j i}$ has the interpretation of a current "donated from WF $j$ to WF $i$." Since $\mathbf{v}_{\langle j i\rangle}=$ $-\mathbf{v}_{\langle i j\rangle}$, the total current carried by any subset of WFs can be computed as the sum of all $\mathbf{v}_{\langle j i\rangle}$ for which $i$ is inside and $j$ is outside the subset. Applying this to the piece of surface region considered above, Eq. (6) becomes

$$
I_{y}=-\frac{e}{\Delta l} \sum_{s} \sum_{s^{\prime}}^{\prime} v_{\left\langle s, s^{\prime}\right\rangle, y}
$$

Setting the boundary deep enough below the surface to be in a bulk-like region and invoking the exponential localization of the WFs and of derived quantities like $\mathbf{v}_{\langle j i\rangle}$, we can identify $\left|\phi_{s}\right\rangle$ and $\left|\phi_{s^{\prime}}\right\rangle$ with the corresponding bulk WFs. Exploiting translational symmetry, $\mathbf{v}_{\left\langle\mathbf{R}, \mathbf{R}^{\prime}\right\rangle}=\mathbf{v}_{\left\langle 0, \mathbf{R}^{\prime}-\mathbf{R}\right\rangle}$, Eq. (8) becomes

$$
I_{y}=-\frac{e}{\Delta l} \sum_{R_{x}<x_{0}} \sum_{R_{x}^{\prime}>x_{0}}^{\prime} v_{\left\langle 0, \mathbf{R}^{\prime}-\mathbf{R}\right\rangle, y}
$$

where the sum is still restricted to a segment of height $\Delta l$. The number of terms in Eq. (9) having a given value of $\mathbf{R}^{\prime}-\mathbf{R}$ is just $\left(R_{x}^{\prime}-R_{x}\right) \Delta l / A_{0}$ if $\left(R_{x}^{\prime}-R_{x}\right)>0$ and zero otherwise. With a change of summation index, Eq. (9) becomes

$$
I_{y}=-\frac{e}{2 A_{0}} \sum_{\mathbf{R}} R_{x} v_{\langle 0, \mathbf{R}\rangle, y},
$$

where the factor of 2 enters because the sum has been extended to all $\mathbf{R}$. For a boundary fragment of arbitrary orientation, Eq. (10) generalizes to $I_{\alpha}=\sum_{\beta} G_{\alpha \beta} \hat{n}_{\beta}$, where $\hat{\mathbf{n}}$ is the unit normal to the boundary, and

$$
G_{\alpha \beta}=-\frac{e}{A_{0} \hbar} \sum_{\mathbf{R}} \operatorname{Im}\left\langle\mathbf{R}\left|r_{\alpha}\right| \mathbf{0}\right\rangle\langle\mathbf{0}|H| \mathbf{R}\rangle R_{\beta} .
$$

The contribution of this itinerant current to the magnetic moment $(1 / 2 c) \oint(\mathbf{r} \times \mathbf{I}) d l$ is easily seen to be related to the antisymmetric part [18] of $G$, so that

$$
M_{\mathrm{IC}}=\frac{1}{c} G_{x y}^{A}=\frac{1}{2 c}\left(G_{y x}-G_{x y}\right) .
$$

Equations (11) and (12) constitute our first major result, showing that the itinerant circulation contribution to the orbital magnetization can indeed be expressed as a bulk property in terms of the bulk WFs alone.

In the remainder of this Letter, we show that the two contributions $M_{\mathrm{LC}}$ and $M_{\mathrm{IC}}$ can both be converted into $\mathrm{k}$-space expressions that can be evaluated directly in the Bloch representation. The WFs are defined via

$$
|\mathbf{R}\rangle=\frac{A_{0}}{(2 \pi)^{2}} \int d^{2} k e^{i \mathbf{k} \cdot(\mathbf{r}-\mathbf{R})}\left|u_{\mathbf{k}}\right\rangle,
$$

where $\left|u_{\mathbf{k}}\right\rangle=e^{-i \mathbf{k} \cdot \mathbf{r}}\left|\psi_{\mathbf{k}}\right\rangle$ is the cell-periodic part of the Bloch function $\left|\psi_{\mathbf{k}}\right\rangle$. Inserting Eq. (1) into Eq. (4) and using $\mathbf{r} \times \mathbf{r}=0$, it follows that

$$
M_{\mathrm{LC}}=\frac{e}{2 A_{0} \hbar c} \operatorname{Im}\langle\mathbf{0}|\mathbf{r} \times H \mathbf{r}| \mathbf{0}\rangle .
$$

Defining $H_{\mathbf{k}}=e^{-i \mathbf{k} \cdot \mathbf{r}} H e^{i \mathbf{k} \cdot \mathbf{r}}$ and using that

$$
\mathbf{r}|\mathbf{R}\rangle=i \frac{A_{0}}{(2 \pi)^{2}} \int d^{2} k e^{i \mathbf{k} \cdot(\mathbf{r}-\mathbf{R})}\left|\partial_{\mathbf{k}} u_{\mathbf{k}}\right\rangle,
$$

Eq. (14) becomes

$$
M_{\mathrm{LC}}=\frac{e}{2 \hbar c} \operatorname{Im} \int \frac{d^{2} k}{(2 \pi)^{2}}\left\langle\partial_{\mathbf{k}} u_{\mathbf{k}}\left|\times H_{\mathbf{k}}\right| \partial_{\mathbf{k}} u_{\mathbf{k}}\right\rangle,
$$


in agreement with Ref. [9].

In order to convert $M_{\text {IC }}$ to $\mathrm{k}$-space, we note that the matrix elements appearing in Eq. (11) are given by

$$
\begin{aligned}
\langle\mathbf{0}|\mathbf{r}| \mathbf{R}\rangle & =\frac{A_{0}}{(2 \pi)^{2}} \int d^{2} k \mathbf{A}_{\mathbf{k}} e^{-i \mathbf{k} \cdot \mathbf{R}}, \\
\langle\mathbf{0}|H| \mathbf{R}\rangle & =\frac{A_{0}}{(2 \pi)^{2}} \int d^{2} k E_{\mathbf{k}} e^{-i \mathbf{k} \cdot \mathbf{R}},
\end{aligned}
$$

where the Berry connection $\mathbf{A}_{\mathbf{k}}=i\left\langle u_{\mathbf{k}}\left|\partial_{\mathbf{k}}\right| u_{\mathbf{k}}\right\rangle$ is real and $E_{\mathbf{k}}$ is the band energy. After some algebra including an integration by parts, we find

$$
G_{\alpha \beta}=-\frac{e}{\hbar} \int \frac{d^{2} k}{(2 \pi)^{2}} E_{\mathbf{k}} \partial_{k_{\beta}} A_{\mathbf{k} \alpha} .
$$

Inserting in Eq. (12) gives

$$
M_{\mathrm{IC}}=-\frac{e}{2 \hbar c} \int \frac{d^{2} k}{(2 \pi)^{2}} E_{\mathbf{k}} \Omega_{\mathbf{k}}
$$

where $\Omega_{\mathbf{k}}=\partial_{\mathbf{k}} \times \mathbf{A}_{\mathbf{k}}$ is the Berry curvature.

Interestingly, both magnetization contributions (16) and (20) are individually gauge-invariant, i.e., insensitive to the choice of phases of the Bloch functions used to construct the WFs. This was shown for Eq. (16) in Ref. [9], and it follows immediately for Eq. (20) because $\Omega(\mathbf{k})$ is a gauge-invariant quantity. Each contribution is also invariant with respect to a shift of the zero of the Hamiltonian; the effect of such a shift is proportional to $\int d^{2} k \Omega_{\mathbf{k}}=2 \pi C$, where $C$ is the Chern number which has been assumed to vanish.

Adding $M_{\mathrm{LC}}$ and $M_{\mathrm{IC}}$, placing both in a common form, and returning to three dimensions, we find that the total magnetization of the crystalline solid can be expressed as

$$
\mathbf{M}=\frac{e}{2 \hbar c} \operatorname{Im} \int \frac{d^{3} k}{(2 \pi)^{3}}\left\langle\partial_{\mathbf{k}} u_{\mathbf{k}}\left|\times\left(H_{\mathbf{k}}+E_{\mathbf{k}}\right)\right| \partial_{\mathbf{k}} u_{\mathbf{k}}\right\rangle
$$

This is our principal result. We note that Eq. (21) is consistent with Eq. (11) of Ref. [10], thereby providing a direct, fully quantum derivation of a result inferred there on the basis of semiclassical arguments alone.

To confirm the correctness of our formulation, we used discretized [9, 19] versions of Eqs. (16) and (20) to calculate $M_{\mathrm{LC}}, M_{\mathrm{IC}}$, and $M$ for the Haldane model using a $300 \times 300 \mathrm{k}$-point mesh. The results, drawn as the lines in Fig. 2, are entirely consistent with the results for finite samples. We also carried out numerical tests which confirmed that the extrapolation of the results from $N \times N$ finite samples for $N=10,20$ and 30 to $N \rightarrow \infty$ shows very precise agreement with the $\mathrm{k}$-space calculation on a dense mesh. We can thus be confident that the formal derivations are correct and that there is no longer any possibility that terms in the magnetization are being overlooked. We have carried out similar tests for other tight-binding models with reduced symmetry [17], with similar results.
In conclusion, we have derived a formula for the orbital magnetization of a crystalline system by working in the Wannier representation, and we have demonstrated its correctness via numerical tests. While limited to the case of a non-interacting zero-Chern-number insulator in a vanishing (or commensurate) magnetic field, our result nonetheless represents significant progress towards a more general theory of orbital magnetization. The resulting formula is easily evaluated in the context of a k-space electronic-structure code. The generalization to the multiband case will be discussed in a forthcoming publication. It remains tantalizingly uncertain whether such a Wannier-based approach can also be generalized to handle insulators with non-zero Chern numbers, metals, or arbitrary magnetic fields.

This work was supported by ONR grant N00014-03-10570 and NSF grant DMR-0233925.

[1] Physics Today 58, 17 (February 2005).

[2] F. Mauri and S. G. Louie, Phys. Rev. Lett. 76, 4246 (1996).

[3] D. Sebastiani and M. Parrinello, J. Phys. Chem. A 105, 1951 (2001).

[4] F. Mauri, B. G. Pfrommer, and S. G. Louie, Phys. Rev. Lett. 77, 5300 (1996); C. J. Pickard and F. Mauri, Phys. Rev. Lett. 88, 086403 (2002).

[5] D. Sebastiani, G. Goward, I. Schnell, and M. Parrinello, Computer Phys. Commun. 147, 707 (2002).

[6] L. L. Hirst, Rev. Mod. Phys. 69, 607 (1997).

[7] R. D. King-Smith and D. Vanderbilt, Phys. Rev. B 47, R1651 (1993); D. Vanderbilt and R. D. King-Smith, Phys. Rev. B 48, 4442 (1993).

[8] R. Resta, Rev. Mod. Phys. 66, 899 (1994).

[9] R. Resta, D. Ceresoli, T. Thonhauser, and D. Vanderbilt, ChemPhysChem, in press.

[10] D. Xiao, J. Shi, and Q. Niu, http://arxiv.org/abs/condmat/0502340.

[11] F. D. M. Haldane, Phys. Rev. Lett 61, 2015 (1988).

[12] K. Ohgushi, S. Murakami, and N. Nagaosa, Phys. Rev. B 62, R6065 (2000).

[13] T. Jungwirth, Q. Niu, and A. H MacDonald, Phys. Rev. Lett. 88, 207208 (2002).

[14] S. Murakami, N. Nagaosa, and S.-C. Zhang, Science 301, 1348 (2003).

[15] Y. Yao, L. Kleinman, A. H. MacDonald, J. Sinova, T. Jungwirth, D.-S. Wang, E. Wang, and Q. Niu, Phys. Rev. Lett. 92, 037204 (2004).

[16] This choice of parameters results in a zero Chern number.

[17] The antisymmetry of the total magnetization $M$ about $\varphi=\pi / 2$ in Fig. 2 is related to the presence of a subtle particle-hole symmetry in the Haldane model $[9,11]$.

[18] The corresponding symmetric part $G_{x y}^{S}$ is related to the time derivative of the spread of Wannier functions [20].

[19] N. Sai, K. M. Rabe, and D. Vanderbilt, Phys. Rev. B 66, 104108 (2002).

[20] N. Marzari and D. Vanderbilt, Phys. Rev. B 56, 12847 (1997). 